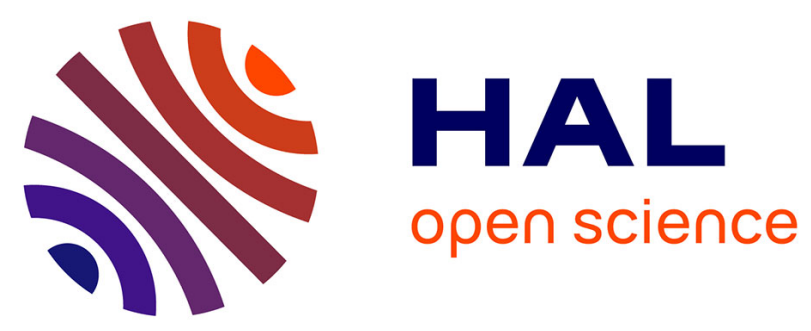

\title{
Finite element simulations of the active stress in the imaginal disc of the Drosophila Melanogaster
}

\author{
V. Pettinati, D. Ambrosi, P. Ciarletta, S. Pezzuto
}

\section{To cite this version:}

V. Pettinati, D. Ambrosi, P. Ciarletta, S. Pezzuto. Finite element simulations of the active stress in the imaginal disc of the Drosophila Melanogaster. Computer Methods in Biomechanics and Biomedical Engineering, 2016, 19 (12), pp.1241-1253. 10.1080/10255842.2015.1124270 . hal-01266290

\section{HAL Id: hal-01266290 \\ https://hal.sorbonne-universite.fr/hal-01266290}

Submitted on 2 Feb 2016

HAL is a multi-disciplinary open access archive for the deposit and dissemination of scientific research documents, whether they are published or not. The documents may come from teaching and research institutions in France or abroad, or from public or private research centers.
L'archive ouverte pluridisciplinaire HAL, est destinée au dépôt et à la diffusion de documents scientifiques de niveau recherche, publiés ou non, émanant des établissements d'enseignement et de recherche français ou étrangers, des laboratoires publics ou privés. 


\title{
ARTICLE \\ Finite element simulations of the active stress in the imaginal disc of the Drosophila Melanogaster
}

\author{
V. Pettinati ${ }^{\mathrm{a}, \mathrm{b}}$, D. Ambrosi ${ }^{\mathrm{a}}$, P. Ciarletta ${ }^{\mathrm{c} *}$, and S. Pezzuto ${ }^{\text {d }}$ \\ ${ }^{a}$ MOX-Politecnico di Milano, piazza Leonardo da Vinci 32, 20133 Milano, Italy; ${ }^{b}$ Fondazione CEN, piazza \\ Leonardo da Vinci 32, 20133 Milano, Italy; ${ }^{c}$ Sorbonne Universités, UPMC Univ Paris 06, CNRS, UMR \\ 7190, Institut Jean Le Rond d'Alembert, F-75005 Paris, France; ${ }^{d}$ Institute of Computational Science, \\ Faculty of Informatics, \\ via Giuseppe Buff 13, 6900 Lugano, Switzerland;
}

\begin{abstract}
During the larval stages of development, the imaginal disc of Drosphila Melanogaster is composed by a monolayer of epithelial cells, which undergo a strain actively produced by the cells themselves. The well-organized collective contraction produces a stress field that seemingly has a double morphogenetic role: it orchestrates the cellular organization towards the macroscopic shape emergence while simultaneously providing a local information on the organ size. Here we perform numerical simulations of such a mechanical control on morphogenesis at a continuum level, using a three-dimensional finite model that accounts for the active cell contraction. The numerical model is able to reproduce the (few) known qualitative characteristics of the tensional patterns within the imaginal disc of the fruit fly. The computed stress components slightly deviate from planarity, thus confirming the previous theoretical assumptions of a nonlinear elastic analytical model, and enforcing the hypothesis that the spatial variation of the mechanical stress may act as a size regulating signal that locally scales with the global dimension of the domain.
\end{abstract}

Keywords: morphogenesis, active strain, finite element analysis, mechano-biology)

\section{Introduction}

The Drosophila Melanogaster, commonly known as "fruit fly", has been extensively studied over the last decades as a system model in developmental biology. The Drosophila is an ideal model organism in developmental biology for several reasons: it is easy to grow in laboratory and its generation time is very short as at a temperature of $28^{\circ} \mathrm{C}$ its development lasts only seven days. Moreover, its females lay up to 100 eggs per day and its complete genome sequence has been completely identified. The embryonal stages take one day, then embryo hatches as first instar larva and, after another 25 hours, molts into a larger wormlike form, the second instar larva. One day later, the second instar larva molts into the third instar larva, the largest of the larval forms, which molts into a pupa after 30 hours. During the pupal stage, the larva is metamorphosing into the adult fly, also called the imago. The Drosophila life cycle is represented in figure 1 . In this work we focus on the third larval stage, and our model refers to the larval organism that develops into the wing of the adult insect: the wing imaginal disc. Morphogenetic events later control cell division, death and rearrangement in a spatially coordinated manner, giving rise to the three-dimensional (3D) adult wing, which is a folded structure with a macroscopic size, eventually made of several thousands of epithelial cells.

\footnotetext{
* Corresponding author. Email: pasquale.ciarletta@upmc.fr
} 
The control in shape and size in the imaginal disc is still a very debated matter among biologists. In particular, it has not yet been fully understood which communication mechanisms locally convey the global information about the disc size, so that the individual cells can adjust and coordinate their mitotic rate during the larval development.

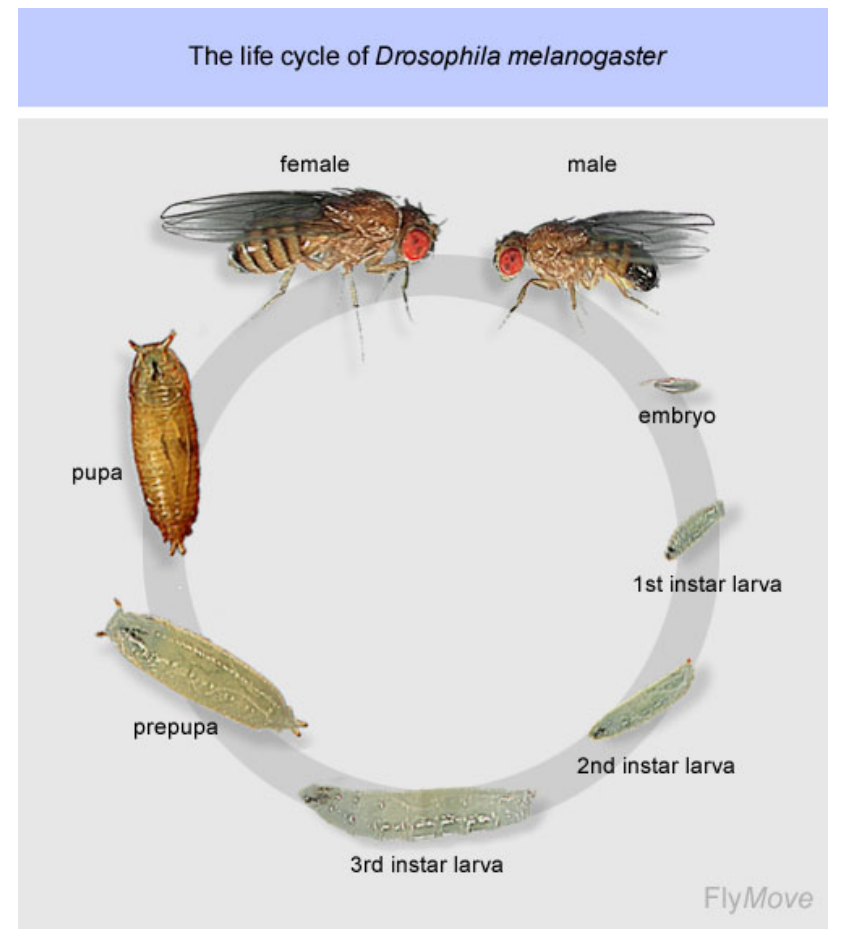

Figure 1. Life cycle of the Drosophila Melanogaster. Figure taken from Weigmann et al (2003)

Morphogens are soluble factors produced by the cells themselves which diffuse and degrade at finite rates and many examples have been identified inside the imaginal disc. Such morphogens have been considered as natural candidates for size control, since an abnormal growth pattern is observed in the wing if their expression is somehow inhibited or unregulated. Notwithstanding, their ability to convey the global information of the size of the wing is controversial: in fact, they are characterized by a short-range action and, most importantly, in the observed reaction-diffusion dynamics neither their concentration nor their concentration gradient invariably scale with size. In other words, if the pattern of morphogen concentration is invariant with the size of the disc, global information cannot be locally transduced.

A possible mechanism of size control that enforces the due scaling characteristics has been proposed by Wartlick and coworkers (Wartlick et al 2011b), who focused on the distribution within the wing imaginal disc of a particular morphogen, the Decapentaplegic (Dpp), in the third larval stage. Wartlick's model is based on two experimental evidences:

- The Dpp distribution is described by a reaction-diffusion equation and its spatial dynamics is fast with respect to the growth of the disc: both diffusion and decay of Dpp can be assumed to be always in mutual equilibrium so that the mass convection due to the material displacement caused by the cell duplication process does not affect the morphogen concentration field $c(x, t)$.

- The cell duplication rate is homogeneous in space.

These experimental observations clearly highlight the conundrum to conciliate an inhomogeneous morphogen distribution with a homogeneous growth: if the concentration of morphogen diffuses with a constant diffusivity and decays at a fixed rate, it cannot scale with the organ size. Thus, Wartlick and coworkers conjectured the existence of a non-degrading molecule that gets diluted 
during the cell proliferation. If the degradation rate of Dpp depends linearly on this molecule, the morphogen concentration might scale as the length of the domain and could possibly trigger a homogeneous growth. Even if other growing control mechanisms have been proposed only relying on the concentration of chemical species (as an example, see Ben-Zvi and Barkai (2010)), a complete explanation of the size determination of the adult wing uniquely in terms of reactive-diffusive agents still remains elusive.

Another possible control mechanism relies on the experimental evidence that the epithelial cells are in a tensional mechanical state during this larval stage, which is generated not by external loads but by some shape changes of the cells themselves. Hufnagel and coworkers (Hufnagel at al 2007) argued that this mechanical stress might compensate the decay of morphogen concentration in the periphery of the disc, so that a combination of morphogen diffusion and mechanical effects might regulate the disc growth.

In a more recent paper, Ambrosi and coworkers (Ambrosi et al 2015) proposed a mathematical model proving that the mechanical stress could convey local information on global size during the wing morphogenesis. Since the epithelial cells are known to actively deform (Lo et al 2000), the presence of an inhomogeneous active contraction, spatially triggered by the morphogen concentration, was found to give rise to a stress distribution embedding the key information about the size of the domain. According to such a mechanism, all the cells receive the same instructive signal, and therefore they can all display the same behavior and stop their duplication process simultaneously when a certain threshold is reached.

In a mathematically formal way, let us state that a generic (chemical, mechanical) signal $f$ can orchestrate the cellular mitosis over a macroscopic affine transformation if it depends on the principal spatial coordinate in the following form:

$$
f(R, t)=f_{0}\left(R_{0}\right) f_{1}\left(R / R_{0}\right),
$$

where $R$ and $t$ denote the spatial and temporal variables respectively and $R_{0}=R_{0}(t)$ stands for the macroscopic length of the domain. In this scenario, the scalar function $f_{0}$ provides the absolute information of the domain size, while the self-similar scalar function $f_{1}$ is constant for a given individual cell because of the homogeneous growth process.

Thus, using a nonlinear elastic model and assuming a two-dimensional (2D) plane strain-plane stress assumption, it has been shown that prescribing an active contraction in an inner circular portion of the imaginal disc (possibly dictated by the higher concentration of morphogens), the elastic equilibrium imposes a stress distribution with derivative of the form given by (1) (Ambrosi et al 2015). The theoretical results are supported by observations. An active contraction has been experimentally reported in the circumferential direction (Mao et al 2011; Nienhaus et al 2009), the stress is compressive in the very central region, and grows in each fixed spatial position as the disc becomes larger.

In this work we corroborate and extend this idea of mechanical transduction to the case of a fully 3D domain by applying a novel numerical simulation tool. We adopt a finite element code to determine the stress pattern induced by an inhomogeneous active contraction in a 3D disc. We consider both an illustrative geometry, made of a thin circular plate, and the real shape of the wing imaginal disc. The numerical simulations allow us to explore contractility patterns with increasing complexity: both radial and biaxial configurations in the morphogens' concentration can be set on the basis of line sources possibly determining the active region. The flexibility of the proposed numerical tool allows us to enforce different symmetries to take into account the experimental reports obtained by different techniques. In particular, we address both the configuration considered by Nienhaus et al. (Nienhaus et al 2009) and Mao et al. (Mao et al 2011), who suggest a radial symmetry in the active strain, and the bilateral configuration reported by Landsberg et al. (Landsberg et al 2009). In both cases, we validate numerically in a 3D geometry the simplifying assumptions that are at the basis of the previous $2 \mathrm{D}$ analytical results.

The paper is organized as follows. Basic notions of continuum mechanics are resumed in section 2 . 
The well-posedness of the mathematical problem and the numerical method are illustrated in section 4 . Sections 3 and 5 are devoted to the analytical determination and numerical approximation of the mechanical signal which conveys the information about the length of the domain in the case of a 2D and 3D domain, respectively. The three-dimensional geometries considered in section 5 are a thin plane and then the real shape of the wing imaginal disc. The numerical results on the strain and stress patterns obtained are finally collected and discussed in section 6 , together with some conclusive remarks.

\section{Kinematics and constitutive assumptions}

Let us consider the imaginal disc as a continuous body $\mathcal{B}$ initially occupying a spatial domain $\Omega_{0} \subseteq$ $\mathbb{R}^{3}$ in the reference configuration. The position vector in the reference configuration is indicated by the material vector $\boldsymbol{X}$. Let $\boldsymbol{\varphi}$ denote the motion function that describes the deformation between $\Omega_{0}$ and the current configuration $\Omega$, so that the spatial position vector is given by:

$$
\boldsymbol{x}=\boldsymbol{\varphi}(\boldsymbol{X}, t),
$$

therefore the displacement field reads:

$$
\boldsymbol{u}(\boldsymbol{X}, t)=\boldsymbol{x}-\boldsymbol{X}=\boldsymbol{\varphi}(\boldsymbol{X}, t)-\boldsymbol{X} .
$$

The deformation gradient tensor $\mathrm{F}$ is the material gradient of the motion:

$$
\mathrm{F}(\boldsymbol{X}, t)=\frac{\partial \varphi(\boldsymbol{X}, t)}{\partial \boldsymbol{X}}=\operatorname{Grad} \boldsymbol{x}(\boldsymbol{X}, t)=\mathbf{I}+\operatorname{Grad} \boldsymbol{u}
$$

where I is the identity matrix, and

$$
J(\boldsymbol{X}, t)=\operatorname{det} \mathbf{F}(\boldsymbol{X}, t),
$$

is the determinant of the Jacobian of the transformation, describing the change from the infinitesimal volume $\mathrm{d} \boldsymbol{X}$ in the reference configuration to the infinitesimal volume in the actual configuration $J(\boldsymbol{X}, t) \mathrm{d} \boldsymbol{x}$.

Since the epithelial cells are mainly composed by water, the incompressibility constraint applies:

$$
J=1 \quad \text { for every } \boldsymbol{X} \in \Omega_{0} .
$$

In nonlinear elasticity, it is useful to describe the deformation introducing the left Cauchy-Green tensor C, the right Cauchy-Green tensor B and the Green strain tensor E, defined as:

$$
\mathrm{C}=\mathrm{F}^{\mathrm{T}} \mathrm{F}, \quad \mathrm{B}=\mathrm{FF}^{\mathrm{T}}, \quad \mathrm{E}=\frac{1}{2}(\mathrm{C}-\mathrm{I}) .
$$

The local balance of linear and angular momentum, in the actual configuration and in absence of external forces and inertia, rewrites:

$$
\left\{\begin{array}{l}
\operatorname{div} \mathrm{T}=0, \quad \text { for } \boldsymbol{x} \in \Omega, \\
\mathrm{T}=\mathrm{T}^{T}, \\
\mathrm{~T} \boldsymbol{n}=0, \quad \text { on } \Gamma_{\mathrm{N}},
\end{array}\right.
$$

where div is the spatial divergence operator, $T$ is the Cauchy stress tensor, $\Gamma_{N}$ is the traction-free portion of boundary, and $\boldsymbol{n}$ is its outward normal. Using the Piola transformation, the equilibrium 
equations (2) in the material configuration read:

$$
\begin{cases}\text { Div FS }=0, & \text { for } \boldsymbol{X} \in \Omega_{0}, \\ F S N=0, & \text { for } \boldsymbol{X} \in \Gamma_{\mathrm{N}}\end{cases}
$$

where Div is the material divergence operator, $\boldsymbol{N}$ is the outward normal in the reference configuration, and $\mathrm{S}$ is the second Piola-Kirchhoff tensor, defined as:

$$
\mathrm{S}=J \mathrm{~F}^{-1} \mathrm{TF}^{-\mathrm{T}}
$$

The disc will be considered as an hyperelastic body, so that the second Piola-Kirchhoff tensor can be described by the means of a scalar strain-energy density function $\mathcal{W}: \Omega_{0} \times \operatorname{Lin}^{+} \rightarrow \mathbb{R}^{+}$, such that:

$$
\mathrm{S}=2 \frac{\partial \widehat{\mathcal{W}}}{\partial \mathrm{C}}
$$

where $\widehat{\mathcal{W}}(\boldsymbol{X}, \mathrm{C})=\widehat{\mathcal{W}}\left(\boldsymbol{X}, \mathrm{F}^{\top} \mathrm{F}\right)=\mathcal{W}(\boldsymbol{X}, \mathrm{F})$. We notice that $\mathrm{S}$ is symmetric, since $\mathrm{C}$ is symmetric.

\section{The axial-symmetric $2 \mathrm{D}$ approximation: analytical results}

The radially symmetric shape of the wing imaginal disc suggests to represent it in first approximation as a circular disc spanned by a polar system of material coordinates $(R, \Theta)$. The disc can be therefore modelled as a circle of radius $R_{0}$, so that the displacement field $\boldsymbol{u}$ has radial component only: $u=u(R)$.

The mechanical driver of this cellular monolayer are not external loads, that are absent, but the active stress produced by the actomyosin cables along the cells boundary (Rauzi et al 2008). The force per unit surface actively produced by the cells is here mathematically encoded in an active strain formulation, formally corresponding to a multiplicative decomposition of the gradient of deformation tensor into two contributions: a passive elastic term $F_{e}$ and an active term $F_{a}$, so that

$$
\mathrm{F}=\mathrm{F}_{\mathrm{e}} \mathrm{F}_{\mathrm{a}}
$$

The active component $F_{a}$ is actively produced by the cells, whilst the hyperelastic strain energy uniquely depends on $F_{e}=F_{a}^{-1}$. The constitutive assumption for $F_{a}$ must be dictated on the basis of the observed strain of the cells in the monolayer, while the passive hyperelastic strain energy $\mathcal{W}\left(\mathrm{F}_{\mathrm{e}}\right)$ should come from standard mechanical tests after myosin inhibition.

We assume that the wing imaginal disc is made of an incompressible isotropic neo-Hookean material, characterized by the following strain-energy function:

$$
\widehat{\mathcal{W}}\left(\mathrm{C}_{\mathrm{e}}\right)=\frac{\mu}{2}\left(\operatorname{tr} \mathrm{C}_{\mathrm{e}}-3\right)=\frac{\mu}{2}\left(\mathrm{C}: \mathrm{C}_{\mathrm{a}}^{-1}-3\right),
$$

where $\mu$ is the shear modulus, $C_{e}=F_{e}^{\top} F_{e}$ and $C_{a}=F_{a}^{\top} F_{a}$.

The active strain $F_{a}$ can be spatially triggered by the morphogens' concentration, which evolves in time following a reaction-diffusion dynamics and typically generates a short-range signal. Let $R_{i} \ll R_{0}$ be the distance from the center of the disc beyond which the morphogen concentration is not anymore detectable by the cells; we suppose that the tissue actively deforms for $R<R_{i}$, while it behaves like inert matter elsewhere. According to some recent experimental observations (Wartlick et al 2011a; Nienhaus et al 2009), we assume that $R_{i}$ scales as $R_{0}$, which implies that the ratio 
$\frac{R_{i}}{R_{0}}$ remains a constant during the whole developmental process. It follows that $\mathrm{F}_{\mathrm{a}}=\mathrm{I}$ for $R>R_{i}$, while it has to be prescribed in a different way according to the observed cell shape distortion in the inner region, which we call the active region.

On the basis of the reported observations (Mao et al 2011), we assume that in polar coordinates $F_{a}$ in the active region is given by

$$
\mathrm{F}_{\mathrm{a}}=\left[\begin{array}{ll}
\delta & 0 \\
0 & \gamma
\end{array}\right]
$$

where $\delta$ and $\gamma$ account for microstructural active strain in the radial and circumferential direction, respectively. The off-diagonal components have been put equal to zero because experimental data do not report a difference in the active contraction over the radial or the circumferential directions. Denoting by $u=r(R)-R$ the radial displacement, we impose the incompressibility constraint $\operatorname{det} F_{e}=1$, and the following interface and boundary conditions for the displacement expressed as a function of the spatial coordinates:

$$
\begin{aligned}
u(0) & =0, & & u\left(r_{i}^{-}\right)=u\left(r_{i}^{+}\right) \\
T_{r r}\left(r_{0}\right) & =0, & & T_{r r}\left(r_{i}^{-}\right)=T_{r r}\left(r_{i}^{+}\right) .
\end{aligned}
$$

Solving the stress balance equations (2), the radial and hoop components of the Cauchy stress can be expressed as a function of the material coordinate $R$ as (Ambrosi et al 2015):

$$
\begin{aligned}
& T_{r r}=T_{r r}\left(R_{i}\right)+\mu\left(\frac{\delta}{\gamma}-\frac{\gamma}{\delta}\right) \log \left(\frac{R}{R_{i}}\right), \\
& T_{\theta \theta}=\mu\left(\frac{\delta}{\gamma}-\frac{\gamma}{\delta}\right)\left(1+\log \left(\frac{R}{R_{i}}\right)\right)+T_{r r}\left(R_{i}\right),
\end{aligned}
$$

for $R \in\left(0, R_{i}\right)$ and

$$
\begin{aligned}
\mathrm{T}_{r r} & =\frac{\mu}{2}\left[\log \left(\frac{\left(R_{0}^{2}+2 \kappa R_{i}^{2}\right)\left(R^{2}+\kappa R_{i}^{2}\right)}{\left(R^{2}+2 \kappa R_{i}^{2}\right)\left(R_{0}^{2}+\kappa R_{i}^{2}\right)}\right)+\kappa R_{i}^{2} \frac{R^{2}-R_{0}^{2}}{\left(R_{0}^{2}+2 \kappa R_{i}^{2}\right)\left(R^{2}+2 \kappa R_{i}^{2}\right)}\right], \\
\mathrm{T}_{\theta \theta} & =\mu\left[\frac{R^{2}+\kappa R_{i}^{2}}{R^{2}}-\frac{R^{2}}{R^{2}+\kappa R_{i}^{2}}+\frac{\kappa}{2} R_{i}^{2} \frac{R^{2}-R_{0}^{2}}{\left(R_{0}^{2}+2 \kappa R_{i}^{2}\right)\left(R^{2}+2 \kappa R_{i}^{2}\right)}\right. \\
& \left.+\frac{1}{2} \log \left(1+\frac{\kappa R_{i}^{2}\left(R^{2}-R_{0}^{2}\right)}{\left(R^{2}+2 \kappa R_{i}^{2}\right)\left(R_{0}^{2}+\kappa R_{i}^{2}\right)}\right)\right],
\end{aligned}
$$

for $R \in\left(R_{i}, R_{0}\right)$, where we have set $\kappa=\gamma \delta-1$.

Since we assume that $R_{i} \ll R_{0}$, the cells in $R>R_{i}$ are subjected to a hoop stress with derivative given by equation (9). For a constant ratio $R_{i} / R_{0}$, the derivative of the hoop stress can be written as:

$$
\frac{d \mathrm{\top}_{\theta \theta}}{d r}=\frac{1}{R_{0}} f\left(\frac{R}{R_{0}}\right)
$$

Both the radial and the hoop stress at each given material point provide an information about $R_{0}$. Nonetheless the radial stress vanishes at the outer boundary, whilsts the hoop stress is almost everywhere and is larger in magnitude and might therefore be a more suitable candidate to orchestrate the homogeneous growth of the disc. The stress field in equations (6)-(9) are represented in figures 5.2.1 and 3. These results are in agreement with experimental data (Nienhaus et al 2009), 

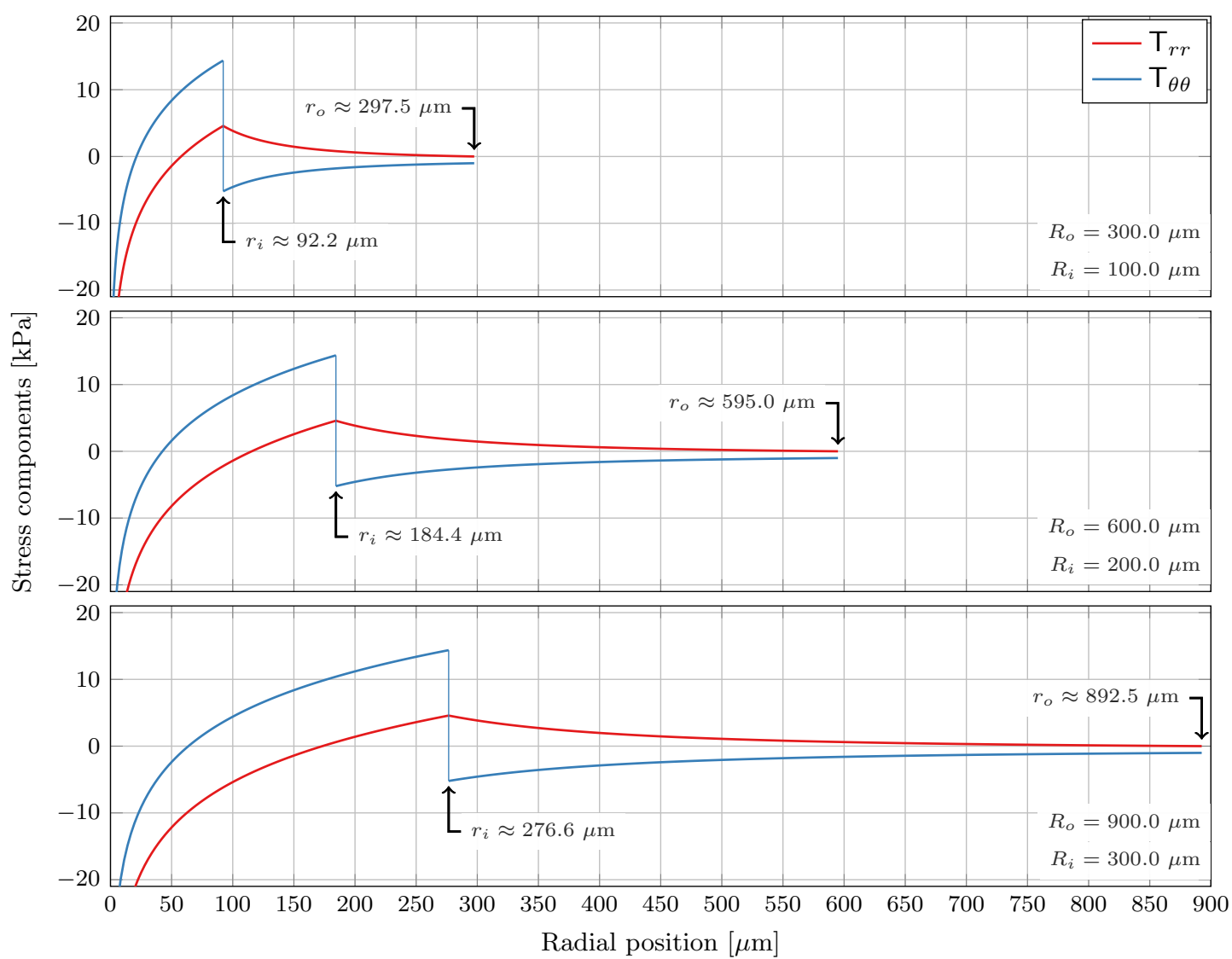

Figure 2. Plot of the radial, circumferential and axial Cauchy stress components versus the actual radial coordinate $r$ at different domain size obtained using a neoHookean strain energy as calculated by a 2D axial-symmetric model. Material parameters are $\mu=30 \mathrm{kPa}, \delta=1$ and $\gamma=0.85$. As expected, the radial stress is continuous, while the hoop stress jumps across the interface $r_{i}$ and changes sign (from compressive to tensional).

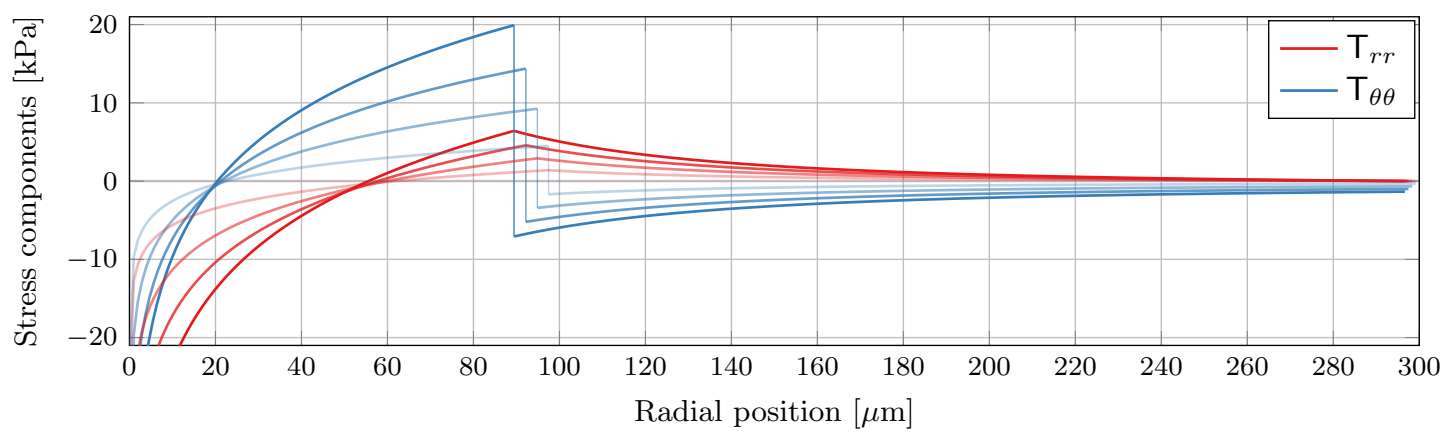

Figure 3. Plot of the radial and circumferential Cauchy stress components as calculated by a 2D axial-symmetric model, versus the actual radial coordinate $r$ at different values of the parameter $\gamma$, corresponding to different intensity of the cell contraction. Material parameters are $\mu=30 \mathrm{kPa}$ and $\delta=1$. The lower the value of $\gamma$, the higher the opaqueness of the curve. Notice that the interface is located at fixed $R_{i}=100$, but the spatial coordinate of the interfce $r_{i}\left(R_{i}\right)$ moves to the left for growing contractility

showing how high stress values arise in the central region of the disc, which increase for each fixed position for a growing domain. 


\section{Variational formulation and finite element implementation}

The determination of the 3D stress generated by the active cellular contraction in the real geometry of the imaginal disc can be only tackled using a numerical procedure. In this section we illustrate the basic ingredients of the numerical approximation; the results of the numerical simulations will be discussed in the following section.

For am unloaded hyperelastic body occupying the domain $\Omega_{0}$ in the reference configuration, the determination of the elastic solution of the balance equations (3) is equivalent to minimize the strain-energy functional that constitutively defines the material. The well-known results from the calculus of variations state that, given a properly defined Sobolev space $V$, the problem of finding an admissible displacement $\boldsymbol{u}$ such that the functional

$$
\mathcal{F}(\boldsymbol{u}):=\int_{\Omega_{0}} \widehat{\mathcal{W}}\left(\boldsymbol{X}, \mathrm{C}_{\mathrm{e}}\right) \operatorname{det} \mathrm{F}_{\mathrm{a}} \mathrm{d} \boldsymbol{X}
$$

is stationary can be solved if the functional is quasi-convex (Dacorogna 2008).

The material of the imaginal disc is assumed to obey an incompressible neo-Hookean law, as in equation (5), with active contraction defined by the unit vector $\boldsymbol{f}$,

$$
\mathrm{F}_{\mathrm{a}}=\gamma_{f} \boldsymbol{f} \otimes \boldsymbol{f}+\gamma_{t}(\mathrm{I}-\boldsymbol{f} \otimes \boldsymbol{f})
$$

where $\gamma_{f}$ and $\gamma_{t}$ are the distortion in the principal and transverse direction, respectively. Substituting the latter expression into (5) we obtain:

$$
\widehat{\mathcal{W}}\left(\mathrm{C}_{\mathrm{e}}\right)=\frac{\mu}{2}\left(\operatorname{tr} \mathrm{C}_{\mathrm{e}}-3\right)=\frac{\mu}{2}\left[\frac{1}{\gamma_{f}^{2} \gamma_{t}^{2}}\left(\gamma_{f}^{2} \operatorname{tr} \mathrm{C}+\left(\gamma_{t}^{2}-\gamma_{f}^{2}\right) \mathrm{C} \boldsymbol{f} \cdot \boldsymbol{f}\right)-3\right]
$$

Rank-one convexity (Ambrosi and Pezzuto 2012) and polyconvexity (Jörg and Neff 2003) can be easily shown for this type of strain-energy, as long as $\gamma_{t} \geq \gamma_{f}>0$.

The incompressibility is handled by means of a Lagrangian multiplier $p \in Q \subset \mathrm{L}^{2}\left(\Omega_{0}\right)$ which enforces the constraint $\operatorname{det} \mathrm{F}_{\mathrm{e}}=1$. It is however numerically convenient to rewrite the strain-energy in terms of the tensor

$$
\overline{\mathrm{F}}_{\mathrm{e}}=\left(\operatorname{det} \mathrm{F}_{\mathrm{e}}\right)^{-1 / 3} \mathrm{~F}_{\mathrm{e}}, \quad \overline{\mathrm{C}}_{\mathrm{e}}=\left(\operatorname{det} \mathrm{C}_{\mathrm{e}}\right)^{-1 / 3} \mathrm{C}_{\mathrm{e}}
$$

Although this redefinition has no substantial effect at a continuum level, it is crucial for the numerical discretization, where in general is not possible to enforce the constraint pointwise, thus ensuring that the strain-energy is correctly evaluated everywhere (Bonet and Wood 1997). The modified functional reads:

$$
\mathcal{F}_{\text {inc }}(\boldsymbol{u}, p):=\int_{\Omega_{0}} \overline{\mathcal{W}}_{\mathrm{e}}\left(\boldsymbol{X}, \overline{\mathrm{C}}_{\mathrm{e}}\right) \operatorname{det} \mathrm{F}_{\mathrm{a}} \mathrm{d} \boldsymbol{X}-\int_{\Omega_{0}} p\left(J-\operatorname{det} \mathrm{F}_{\mathrm{a}}\right) \mathrm{d} \boldsymbol{X}
$$

The characterization of the stationary point $(\boldsymbol{u}, p) \in V \times Q$ of (13) is obtained from the first variation of the functional:

$$
\int_{\Omega_{0}} \mathrm{~S}: \mathrm{F}^{\top} \operatorname{Grad} \boldsymbol{\eta} \mathrm{d} \boldsymbol{X}-\int_{\Omega_{0}} q\left(J-J_{\mathrm{a}}\right) \mathrm{d} \boldsymbol{X}=0, \quad \text { for every }(\boldsymbol{\eta}, q) \in V \times Q,
$$

where $J_{\mathrm{a}}=\operatorname{det} \mathrm{F}_{\mathrm{a}}$ and $\mathrm{S}$ is the second Piola-Kirchhoff tensor. Under the assumption (12) the 
Cauchy stress tensor is given by:

$$
\mathrm{T}=\frac{J_{\mathrm{a}}^{5 / 3}}{J} \frac{\mu}{\gamma_{f}^{2} \gamma_{t}^{2}} \operatorname{dev}\left[\gamma_{f}^{2} \overline{\mathrm{B}}+\left(\gamma_{t}^{2}-\gamma_{f}^{2}\right) \overline{\mathrm{B}} \boldsymbol{f} \otimes \boldsymbol{f}\right]-p \mathbf{I}
$$

where $\operatorname{dev}(\cdot)$ is the deviatoric operator, returning the trace-free part of its argument.

The numerical discretization of the variational problem (14) is performed using the finite element method, by selecting finite dimensional subspaces $V_{h}$ and $Q_{h}$ of $V$ and $Q$, respectively. The nonlinear problem is then solved by means of the Newton-Raphson iterative method, which requires the linearized variational problem. We implemented the numerical code in the FENICS framework (Logg et al 2012), with quadratic finite elements for the displacement and linear finite elements for the pressure on a tetrahedral (or triangular) mesh. We impose in numerical simulations that the material point in the origin has zero displacement, also fixing null rotation and no load applied at the boundary. The numerical solution is obtained using 70962 elements and 119130 degrees of freedom for the thin cilinder (section 5.2); 57606 elements and 97150 degrees of freedom have been used for real wing geometry (section 5.3). The results are mesh independent: the typical mesh size $h$ has been selected after comparing the solution with results obtained using $2 h$, which do not exhibit significant differences.

We conclude this section mentioning that our implementation can take advance of possible symmetries of the problem. For instance, in the case of the thin disk, described in the next section, we restrict the problem to the set of axisymmetric solutions of the form:

$$
\left\{\begin{array}{l}
r=R+u_{R}(R, Z), \\
z=Z+u_{Z}(R, Z), \\
\theta=\Theta
\end{array}\right.
$$

where $(r, z, \theta) \in \Omega$ and $(R, Z, \Theta) \in \Omega_{0}$. In this case, the physical components of the deformation gradient tensor are:

$$
\mathrm{F}=\left[\begin{array}{ccc}
1+\frac{\partial u_{R}}{\partial R} & \frac{\partial u_{R}}{\partial Z} & 0 \\
\frac{\partial u_{Z}}{\partial R} & 1+\frac{\partial u_{Z}}{\partial Z} & 0 \\
0 & 0 & \frac{r}{R}
\end{array}\right]
$$

while the variational formulation is modified by substituting $\mathrm{d} \boldsymbol{X}$ with $2 \pi R \mathrm{~d} \boldsymbol{X}$ and integrating only over one section $(R, Z)$ in $\Omega_{0}$.

Finally, the relevant geometric, mechanical and biological parameters implemented in the numerical simulation are collected in Table 4

Table 1. Parameters characterizing the wing imaginal disc in the numerical simulations.

\begin{tabular}{|c|c|c|}
\hline Symbol & Meaning & Value \\
\hline$R_{0}$ & Radius of the cylindrical domain & $450 \mu \mathrm{m}$ \\
\hline$R_{i}$ - cylindrical domain & Radius of the cylindrical active region & $150 \mu \mathrm{m}$ \\
\hline$R_{i}$ - real geometry of the wing disc & Radius of the cylindrical active region & $300 \mu \mathrm{m}$ \\
\hline$h_{0}$ & Thickness of the cylindrical domain & $30 \mu \mathrm{m}$ \\
\hline$\delta$ & Magnitude of the radial active contraction & 1 \\
\hline$\gamma$ & Magnitude of the hoop active contraction & 0.85 \\
\hline$\mu$ & Shear modulus (Schluckl and Agderter 2010) & $30 \mathrm{KPa}$ \\
\hline
\end{tabular}




\section{The 3D imaginal disc: geometry and experimental facts}

The 2D stress field analytically determined in section 3 qualitatively reproduce some key experimental observations, thus supporting the conjecture that the stress variation is a possible communication mechanism for size orchestration. Nevertheless, the two dimensional approximation is to be validated by a quantitative comparison with the results of the $3 \mathrm{D}$ numerical simulations. To assess the quasi two-dimensionlity of the problem, we address different 3D shapes of the active region, with different active contraction patterns: a thin cylinder and the real wing imaginal disc shape. A discussion on the discrepancies between full 3D and 2D axial-symmetric results will support the theory illustrated above.

A reliable 3D numerical simulation of the tensional pattern in the wing imaginal disk and its relationships with the dynamics of morphogenesis is to be based on a precise knowledge of the morphogenetic processes. Even if we are aware of the very complex signaling pathways that occur in the imaginal disc during its development, here we do not address in details such a signaling dynamics. In our work we are interested in the presence of soluble factors only in view of their mechanical role; we therefore restrict the discussion on morphogenetic gradients to the minimum degree of detail necessary for our purpose. In the following paragraph, we summarize the main known features of the morphogens' diffusive dynamics, with a particular focus on the shape and position of the source lines of the soluble factors which generate the symmetries that we exploit in our modeling approach.

\subsection{Sources and patterns of morphogens in the wing imaginal disc}

Three different regions can be identified within the wing imaginal disc: the notum (proximal), the hinge (central) and the pouch (distal), which is represented in figure 4 . The notum will give rise to the torax of the fruit fly, the pouch to the wing and the hinge to the flexible region between the two.

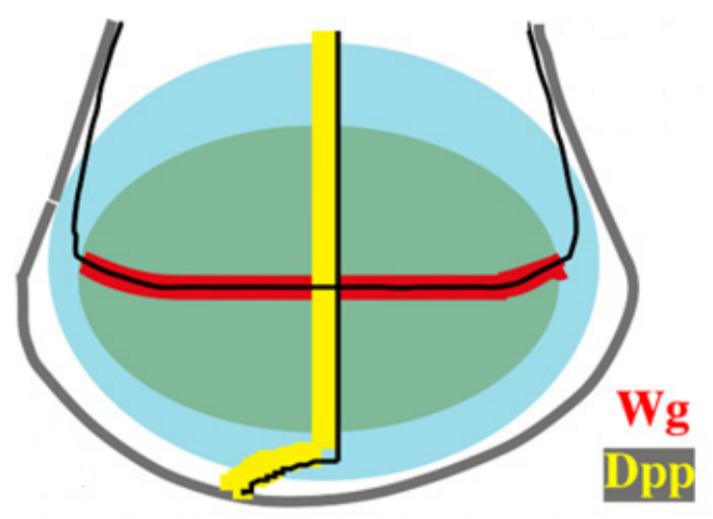

Figure 4. Schematic of a portion of the wing imaginal disc, bounded by the grey line. The grey region represents the wing pouch, from which the wing originates. The Dpp source along the anterior-posterior boundary is marked by the yellow line, while the Wg source along the dorsal-ventral boundary by the red line. Figure adapted with permission from Rogulja et al (2008).

The disc can be described by two orthogonal axes: the anterior-posterior axis, which is a longitudinal line that divides the disc into two almost equal parts, and the dorsal-ventral axis, which crosses the previous one at the center of the wing pouch. Each axis that goes from the center to the perifery of the disc is referred to as proximal-distal axis. It is worth noting that this configuration changes in the adult insect: since during the transition from the larva to the imago the disc folds and becomes bilayered, the original symmetry gets lost. In the adult, the dorsal and ventral regions are defined as the external part of the wing and the one in contact with the fly body, respectively. 
The proximal-distal axis connects the point by which the wing is attached to the body (proximal) to the opposite end (distal), and it is orthogonal to the anterior-posterior boundary.

Among many others, two morphogens play a major role in the developmental biology literature: the Decapentaplegic (Dpp) and the Wingless (Wg). The Dpp source is located along the anteriorposterior boundary. The production rate, the effective diffusion coefficient and the degradation rate of Dpp have been measured experimentally (Kicheva et al 2007). The spatial dynamics of Dpp is fast with respect to the growth of the disc: diffusion and decay of Dpp can be assumed to be always in mutual equilibrium so that the mass convection due to the material displacement caused by the cell duplication process does not distort the morphogen concentration field $c(x, t)$. According to recent studies, the Dpp profile scales in space with the disc length and the morphogen concentration adapts to disc size (Wartlick et al 2011b,a). Notwithstanding, such an experimental observation is criticized by other groups (Hufnagel at al 2007). The biophysical properties of $\mathrm{Wg}$ are less known. Like the Dpp, it is a molecule that diffuses away from a localized source to directly instruct cell identity in a concentration-dependent manner, but its distribution in the wing imaginal disc is not as regular as the one for Dpp. It is expressed in two ring-like domains in the hinge region, along the dorsal-ventral line, which is orthogonal to the anterior-posterior one and passes through the center of the pouch, and in a broad band in the dorsal part of the disc.

According to several authors (Aegerter-Wilmsen et al 2007; Hufnagel at al 2007; Shraiman 2005), the composition in the concentration of Dpp and $\mathrm{Wg}$ in the wing pouch can be identified as the instructive signal that regulates the disc growth, produced at the intersection of the two line sources.

\subsection{Inhomogeneous active strain in a circular disc}

We first consider a simplified model of the imaginal wing disc as a thin circular disc composed by a soft elastic material. The domain $\Omega_{0}$ is therefore initially characterized by radius $R_{0}$ and thickness $h$, respectively, where in a cellular monolayer with thousand of cells $h / R_{o} \lesssim 0.04$. A cylindrical material coordinate system $(R, Z, \Theta)$ is consistently used. In the following, two different symmetries for the morphogenetic control will be discussed.

\subsubsection{Central pointwise source of morphogenetic control}

An active region with cylindrical symmetry is generated by a central pointwise source, possibly located at the intersection between the line sources of Dpp and $\mathrm{Wg}$, as depicted in figure 4. The assumption of a radial symmetry is corroborated by the geometrical distribution of the cells in the wing imaginal disc (Breen et al 2012) and by the presence of atypical myosin Dachs. In fact, according to (Mao et al 2011), the localization of Dachs correlates with the orientation of cell division and tissue growth in the developing fly wing. Being localized at the distal side of each cell's apical surface, the cells grow preferentially along the $\mathrm{P}-\mathrm{D}$ axis, forming elongated shapes before dividing (see figure 5).

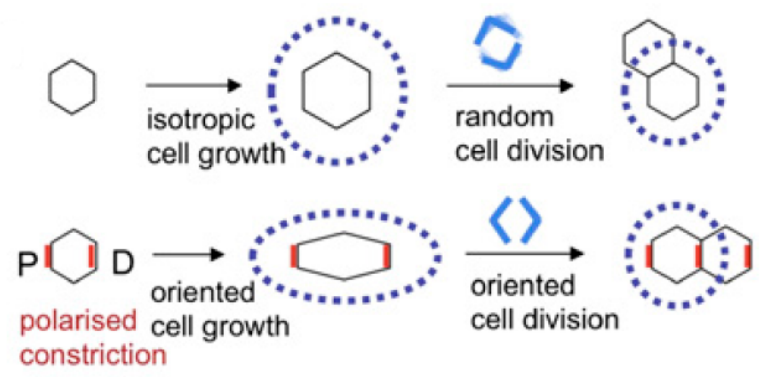

Figure 5. Cell growth, shape, and division in an isotropic (top) and polarized (P-D axis) fashion, generated by Dachs gradient (bottom). Figure adapted with permission from Mao et al (2011).

In the wing of the adult Drosophila, the $\mathrm{P}-\mathrm{D}$ axis is perpendicular to the anterior-posterior axis, 
but during the larval stages it can be approximated as a line that goes from the center to the periphery of the wing imaginal disc, as established by the combined action of signals emanating from the anterior-posterior and the dorsal-ventral compartments (Rogulja et al 2008). In our frame of reference, the $\mathrm{P}-\mathrm{D}$ line is described by the radial axis, therefore the elongation along $\mathrm{P}-\mathrm{D}$, in the presence of an incompressible material, is compatible with a circumferential contraction. In accordance with the experimental studies reporting an active hoop strain of the actomyosin cables, we impose a circumferential compression in an inner portion of the disc within. Thus, we employ a multiplicative decomposition of the deformation gradient tensor $\mathrm{F}$ in the region $R<R_{i}<R_{0}$, whose active component $F_{a}$ rewrites

$$
\mathrm{F}_{\mathrm{a}}=\mathrm{I}+(\gamma-1) \boldsymbol{e}_{\Theta} \otimes \boldsymbol{e}_{\Theta}
$$

with $\gamma \in(0,1]$ and $\boldsymbol{e}_{\Theta}$ is the spatial unit vector in the circumferential direction. For this case $J=\operatorname{det} \mathrm{F}_{\mathrm{a}}=\gamma$ and the Cauchy stress tensor (15) reads:

$$
\mathrm{T}=\mu \operatorname{dev}(\mathrm{B})+\mu \frac{1-\gamma^{2}}{\gamma^{2}} \operatorname{dev}(\mathrm{B}) \boldsymbol{e}_{\Theta} \otimes \boldsymbol{e}_{\Theta}-p \mathrm{I}
$$

For $R>R_{i}$ the cellular material only produces a passive elastic response, i.e. $\gamma=1$, while for $R<R_{i}$ the material is subjected to an active contraction with $\gamma<1$. In $R=R_{i}$ a discontinuity in the material parameters is present. To overcome numerical issues around this sharp interface, we adopt a smoothed $\gamma$ of the form:

$$
\gamma(R)=\frac{1-\gamma}{2}\left[1+\tanh \left(\frac{R-R_{i}}{\varepsilon}\right)\right]+\gamma
$$

and we refine the mesh around $R=R_{i}$ in order to correctly solve the boundary layer.

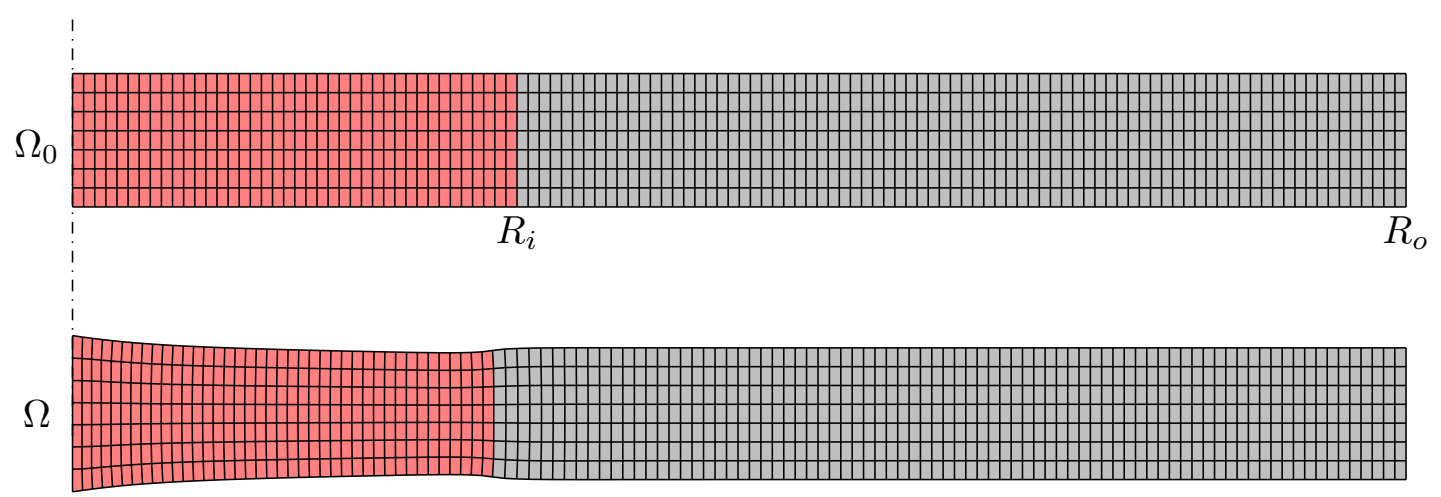

Figure 6. Reference and deformed configuration of a section of the imaginal disc in the $(r, z)$ plane. The red shaded area is the active zone, while the grey area is purely passive. The displacement in the $Z$ direction is small with the respect to the radial deformation. Geometrical and material parameters are $R_{i}=150 \mu \mathrm{m}, R_{o}=3 R_{i}, h=30 \mu \mathrm{m}, \mu=30 \mathrm{kPa}$ and $\gamma=0.85$.

The deformation and stress fields resulting from numerical simulation, performed with $\gamma=0.85$, $R_{i}=150 \mu \mathrm{m}, R_{0}=3 R_{i}$, and $h=30 \mu \mathrm{m}$, are shown in figure 6 and 7 , respectively. Notice that while the assumption $R_{i} \ll R_{0}$ holds, in these figures we adopt $R_{i}=R_{0} / 3$ for a better graphical representation of the results.

The radial and hoop stress provided by 3D numerical simulations are plotted in figure 8 versus the radial coordinate. The results almost overlap the corresponding ones in the $2 \mathrm{D}$ case reported in figure : they are both compressive in the active region, where their magnitude is higher than in the rest of the domain. The axial stress, not resolved by the the $2 \mathrm{D}$ model, is much smaller than the other components, and none of them changes significantly along $z$. These numerical simulations corroborate the results of our previous $2 \mathrm{D}$ model. 

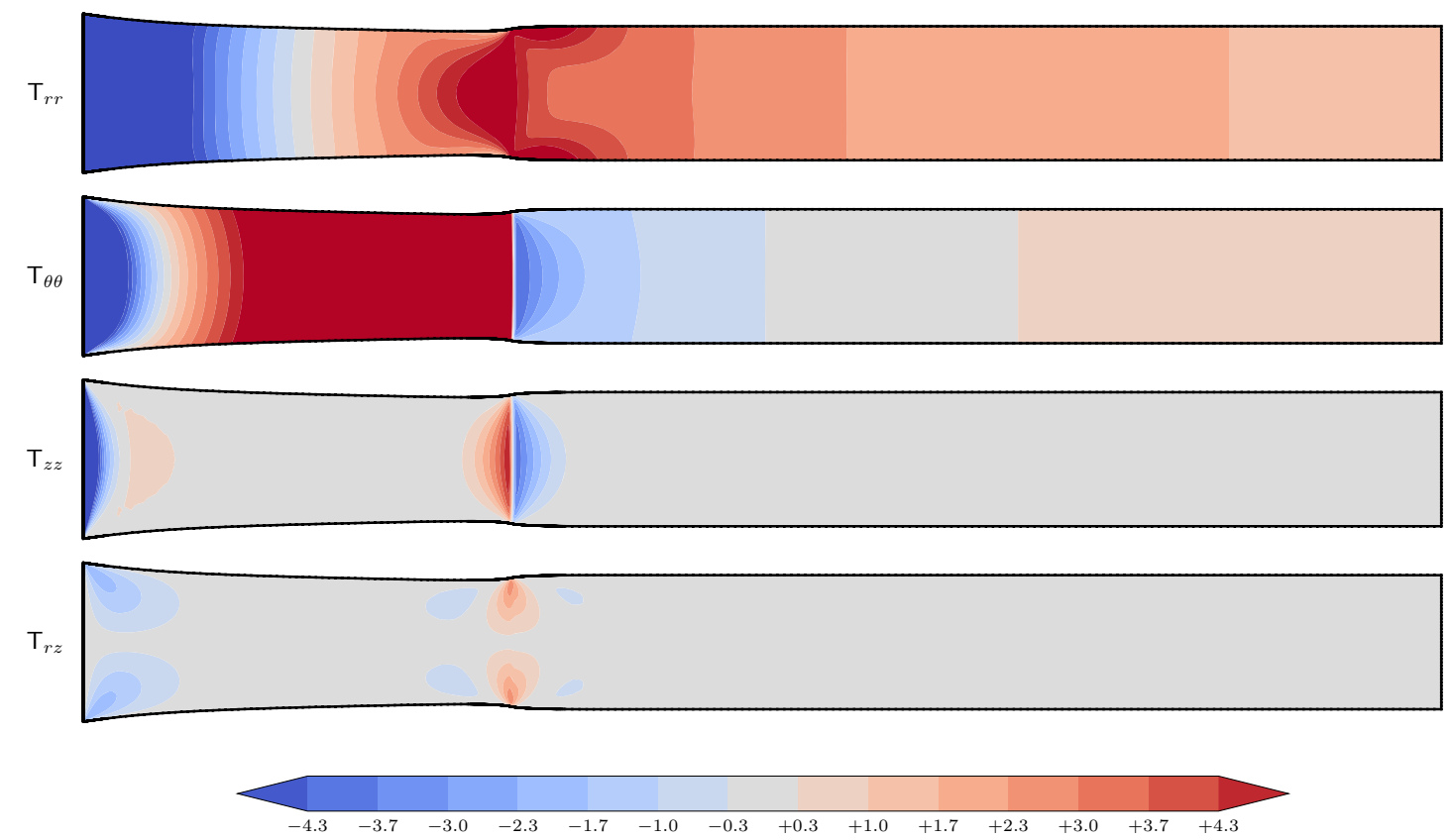

Figure 7. Contour plot of the Cauchy stress components (in $\mathrm{kPa}$ ) across a radial section of the imaginal disc in the $(r, z)$ plane. In the active region, the stress magnitude is higher than in the passive zone, where it rapidly approaches zero while getting closer to the outer boundary. All the components depend weakly on the axial coordinate, $\mathbf{T}_{z z}$ and $\mathrm{T}_{r z}$ are much smaller then the others.

In every fixed point of the material framework, compression (tension) grows as far as the domain grows. This is in agreement with the calculations carried out with the planar model and, most important, with the experimental observations (Nienhaus et al 2009) (see figure 8).

\subsubsection{Line source of morphogenetic control}

Even if we are not able to identify with full confidence the specific morphogens triggering the active contraction, we know something about their patterns, since some of them are produced along lines that have been identified in the wing imaginal disc. These source lines can break the axial symmetry of the problem, so that the concentration field becomes genuinely 3D. This scenario, which is hard if not impossible to be tackled by analytical tools, can be numerically investigated. We therefore assume here that the activating morphogen (possibly Dpp) regulates the tissue growth and concentrates in a thin stripe centered in the anterior-posterior axis, which corresponds to the active region. This setting, which differs from the radially symmetric one illustrated above, is supported by the experimental results of Landsberg and coworkers (Landsberg et al 2009). Using a laser, they cut the disc along different lines and observe the tissue displacement; they estimate that the mechanical tension along the anterior-posterior boundary is approximately 2-5 times bigger than in the rest of the tissue. Using a Cartesian frame of reference $(x, y, z)$ with origin in the center of the disc, such that the disc is symmetric about the plane $z=0$, and representing the anterior-posterior axis by the $y$-axis, the active region is here defined by $|x|<L_{c}$, with $L_{c} \ll R_{0}$. Within this region we impose an active contraction along the $y$-axis. In this case, the tensor that describes the active contraction is expressed in a Cartesian basis and reads

$$
\mathrm{F}_{\mathrm{a}}=\mathrm{I}+(\gamma-1) \boldsymbol{e}_{2} \otimes \boldsymbol{e}_{2}
$$

The computed stress distribution for $\gamma=0.85$ is shown in figure 9 .

This result partially contrasts with photoelasticity measurements where the stress distribution in the disc does not exhibit such a bilateral symmetry. While further experimental data are needed 

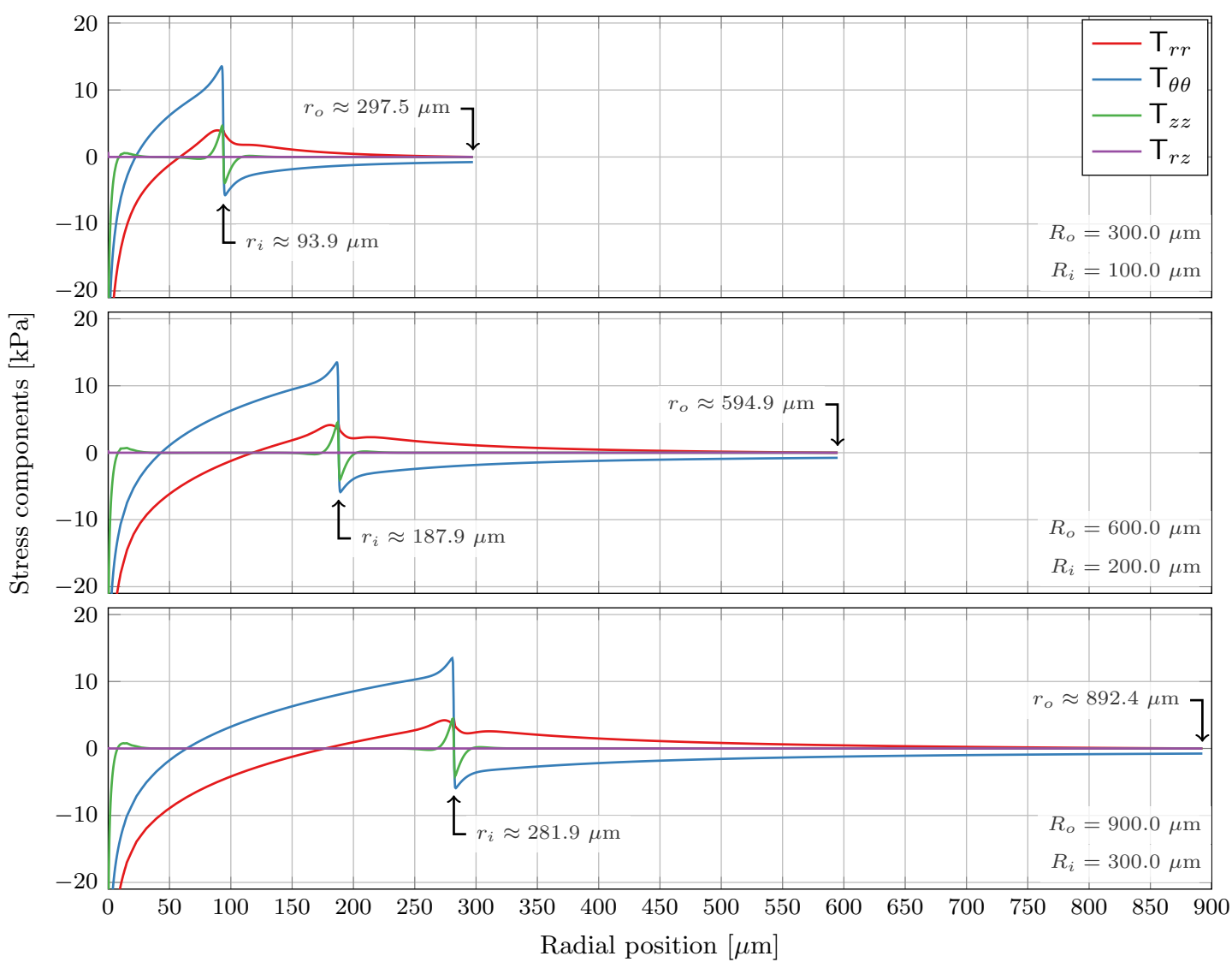

Figure 8. Plot of the Cauchy stress tensor components $\mathrm{T}_{r r}, \mathrm{~T}_{r z}, \mathrm{~T}_{\theta \theta}$ and $\mathrm{T}_{z z}$ versus the radial coordinate at $\theta=0$ and $z=0$. $\mathrm{T}_{\theta \theta}$ moves from compressive to tensile within the active region and it reaches the maximum in $R=R_{i}$, where it undergoes a discontinuity. In the passive region, the magnitude of $\mathrm{T}_{\theta \theta}$ is lower then in the active one, whilst it is everywhere higher than $\mathrm{T}_{z z}$ and $\mathrm{T}_{r r} . \mathrm{T}_{r r}$ has a similar distribution as $\mathrm{T}_{\theta \theta}$, and it approaches zero soon after $R=R_{i}$. The magnitude of $\mathrm{T}_{z z}$ is everywhere much smaller than the other components. Geometrical and material parameters are the same as in Figure 7.

to characterize the stress pattern, we remark that the flexibility of the numerical method is in the ability to incorporate different active region: a central stripe of the disc or with a radially symmetric area in the wing pouch.

\subsection{Real geometry of the wing imaginal disc}

Given the cylindrical symmetry of both the cell arrangement and the stress pattern reported by most experimental observation, the cylindrical domain is a good starting point to model the growing wing imaginal disc. As a next step, in this section we consider the real 3D geometry of the disc, with a cylindrical active region centered in the pouch bounded by the material coordinate $R<R_{i}$. The wing imaginal disc is a thin 3-D structure, whose thickness $h$ is much smaller that the length of the anterior-posterior and dorsal-ventral axes. The origin of the cylindrical system of coordinates is located at the intersection between the anterior-posterior and the dorsal-ventral axes, so that the domain is symmetric above the plane $z=0$. In the region delimited by a radial position $R_{i} \ll R_{0}$ we impose an active contraction as defined in equation (16), while $\mathrm{F}_{\mathrm{a}}=\mathrm{I}$ for $R>R_{i}$. As previously assumed, the material is neo-Hookean and incompressible. We have run our finite element solver to find the solution of the stress balance equation. The results are reported in figures 9 and 10 .

As expected, the magnitude of the stress is considerably higher in the center than in the periphery of the disc and for small values of $R$ the disc is under compression both in radial and hoop components; moreover the magnitude of the $z$-component of the stress is negligible with respect to the others. We can conclude that the results we had obtained with a 2-D domain are able to 

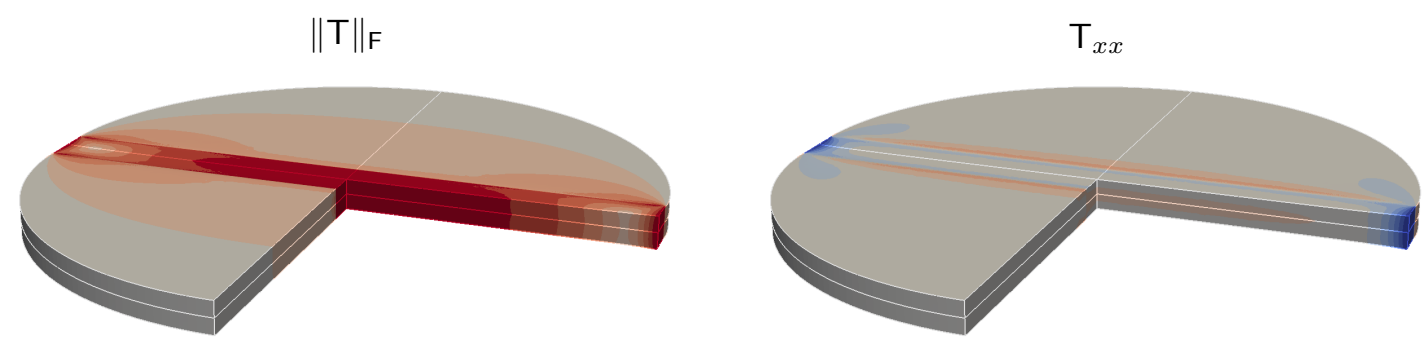

$\mathrm{T}_{y y}$
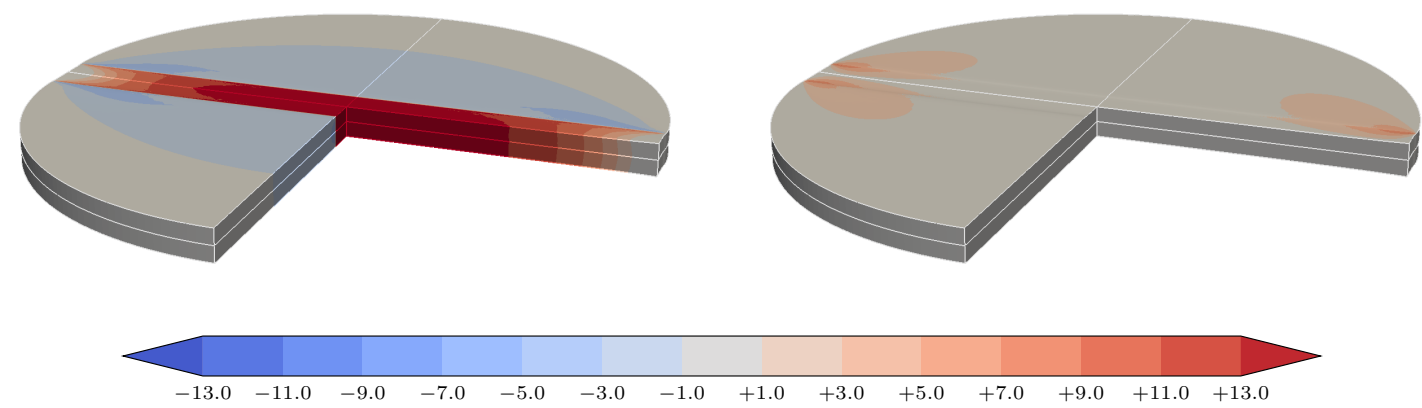

Figure 9. Color plot of the stress distribution (in $\mathrm{kPa}$ ) computed with active contraction triggered by a Dpp concentration threshold. The active region corresponds to a stripe centered in the anterior-posterior axes and whose thickness is taken to be one tenth of the disc diameter. A bilateral symmetry is found in the stress distribution. The disc diameter is $300 \mu \mathrm{m}$ and its thickness is $h=15 \mu \mathrm{m}$. Material parameters are $\mu=30 \mathrm{kPa}$ and $\gamma=0.85$.
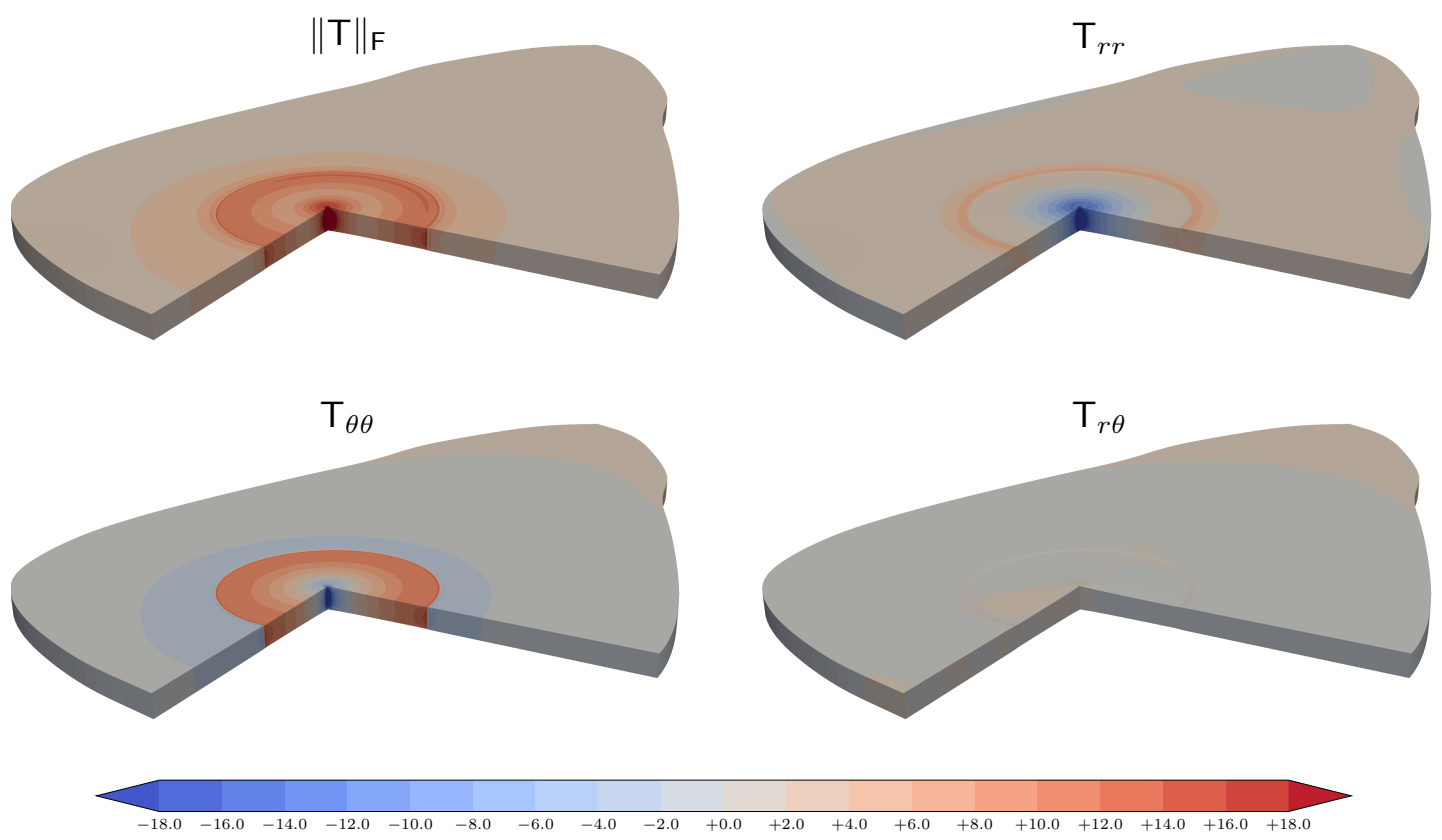

Figure 10. Colour plot of the stress distribution (in $\mathrm{kPa}$ ) for a slice at $z=0$ of a wing-shaped domain. The active contraction occurs in a region with a diameter of $300 \mu \mathrm{m}$. In the active region, the stress magnitude is higher than in the passive one, where it approaches zero. Material parameters are $\mu=30 \mathrm{kPa}$ and $\gamma=0.85$.

qualitatively capture the essential stress behavior of a realistic 3D geometry. The results of our numerical simulations are reported in figures 10, and can be compared with the existing stress measures reported from photoelasticity experiments, which are reproduced in figure 11 and show the distribution of the retardance within the disc: the pattern of the stress obtained experimentally can be qualitatively reproduced by our numerical simulations. 


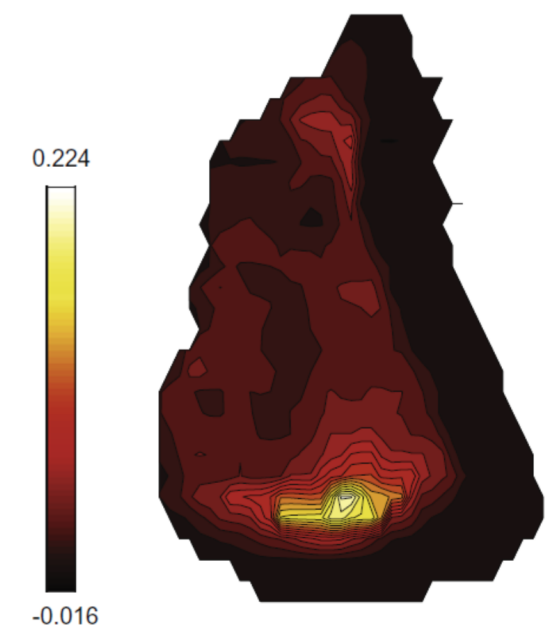

Figure 11. Distribution of retardance in a normal, late stage third instar wing disc. The retardance is highest in the centre of the imaginal wing disc, showing that this region is subjected to the highest compression. Figure taken from Nienhaus et al (2009).

\section{Final remarks}

The 3D stress field produced by the cells contractility in the wing imaginal disc of a fruit fly has been numerically computed using a finite element code under the assumption that the cell monolayer behaves elastically on the time scale of interest.

The first result of this work is a validation of analytical results previously obtained on the basis of an assumption of plane strain. Our simulations confirm that the planar components of the stress in the fully $3 \mathrm{D}$ setting strongly resemble the ones obtained in the $2 \mathrm{D}$ case, thus confirming that the planar axialsymmetric approximation is able to catch the relevant features of the stress distribution. Moreover, the $z$-components of the computed stress is much smaller than the other components and do not significantly vary along the thickness, thus supporting the simplifying $2 \mathrm{D}$ assumptions. The quasi planarity of the solution therefore confirms that the gradient of the stress can be a mechanical signal that transduces information to the cells about the growth of the wing imaginal disc, thus providing a suitable control mechanism on its final size. In particular, we suggest that the hoop stress is the most suitable candidate to regulate the homogeneous growth of the disc, since the radial component rapidly approaches zero in the passive region. This mechanical signal is compatible with the experimentally observed homogeneous growth, since it changes in time for each individual cell as a function of the domain length: when the proper domain size is reached, all the cell receive the same signal and they simultaneously slow down their duplication rate, eventually stopping.

In our 3D numerical simulations the wing imaginal disc is first modeled by a thin cylinder and then represented by its real shape. In both cases the axial stress is negligible compared to the other components and this result validates the analytical solutions obtained in the plane.

The second result of this work is that the numerical simulations reproduce a stress pattern in qualitative agreement with recent experimental observations. The adopted radial symmetry seems to be the most suitable choice at the light of some experimental results (Nienhaus et al 2009; Mao et al 2011), supporting the strategy to approximate analytically the imaginal discs as circles of radii $R_{0}$ and $R_{i}<R_{0}$, respectively. Moreover, the flexibility of the numerical framework allows us to manage different shapes of the active region. In contrast with works that report a stress magnitude that decreases as the distance from the center of the domain increases (Nienhaus et al 2009), some studies suggest a bilateral stress distribution because they reproduce a maximum tension along the anterior-posterior boundary (Landsberg et al 2009).

The mathematical model contains a very limited number of material and biological parameters (the shear modulus, the micro-strain ratio, the radius of the active region). More experimental data 
are definitely needed to determine the values of the stress components in the wing imaginal disc, however our model seems encouraging for its flexibility in incorporating further mechanobiological information as soon as available. Although the affine transformation of the imaginal disc is a specific biological system, the proposed numerical method may be relevant for other growth problems in living matter, since it can be easily refined in order to couple growth, active contraction and diffusing signals/biomolecules in a fully $3 \mathrm{D}$ environment.

\section{References}

Aegerter-Wilmsen T, Aegerter CM, Hafen E and Basler K, Model for the regulation of size in the wing imaginal disc of Drosophila, Mech. Dev. 124: 318-326 (2007).

Ambrosi D and Pezzuto S (2012) Active stress vs. active strain in mechanobiology: constitutive issues, J. Elasticity, 107(2):199-212.

Ambrosi D, Pettinati V and Ciarletta P (2015) Active stress as a local regulator of global size in morphogenesis. International Journal of Nonlinear Mechanics (in press, doi:10.1016/j.ijnonlinmec.2014.11.027).

Ben-Zvi D and Barkai N (2010) Scaling of morphogen gradients by an expansion-repression integral feedback control Proc. Natl Acad. Sci. USA, 107: 6924-6929.

Bonet J and Wood RD (1997) Nonlinear continuum mechanics for finite element analysis.

Breen D, Widmann T, Bai L, Jülicher F, Dahmann C (2010) Epithelial Cell Reconstruction and Visualization of the Developing Drosophila Wing Imaginal Disc, IEEE Symposium on Biological Data Visualization (BioVis).

Dacorogna B (2008) Direct Methods in the Calculus of Variation, Springer.

Hufnagel L, Teleman AA, Rouault H, Cohen SM and Shraiman BI (2007) On the mechanism of wing size determination in fly development, Proc. Natl Acad. Sci. USA, 104:3835-3840

Jörg S and Neff P (2003) Invariant formulation of hyperelastic transverse isotropy based on polyconvex free energy functions, Int. J. Sol. Struct. 40.2:401-445.

Kicheva A, Pantazis P, Bollenbach T, Kalaidzidis Y, Bittig T, Jülicher F and Gonzalez-Gaitan M (2007) Kinetics of morphogen gradient formation. Science, 315: 521-525

Landsberg KP, Farhadifar R, Ranft J, Umetsu D, Widmann TJ, Bittig T, Said A, Jülicher F and Dahmann C (2009) Increased cell bond tension governs cell sorting at the Drosophila anteroposterior compartment boundary, Current Biology 19:1950-1955.

Rauzi M, Verant P, Lecuit T and Lenne PF (2008) Nature and anisotropy of cortical forces orienting Drosophila tissue morphogenesis. Nat Cell Biol. Dec;10(12):1401-10.

Lo CM, Wang HB, Dembo M and Wang YL (2000) Cell movement is guided by the rigidity of the substrate, Biophys. J., 79(1):144-152

Logg A, Wells GW and Hake J (2012) DOLFIN: A C++/Python finite element library. Springer Berlin Heidelberg.

Mao Y, Tournier AL, Bates PA, Gale JE, Tapon N and Thompson BJ (2011) Planar polarization of the atypical myosin Dachs orients cell division in Drosophila, Genes and Development, 25:131-136.

Nienhaus U, Aegerter-Wilmsen T and Aegerter CM (2009), Determination of mechanical stress distribution in Drosophila wing disc using photoelasticity, Mechanics of Development, 126:942-949

Pezzuto S, Ambrosi D and Quarteroni A (2014) An orthotropic active-strain model for the myocardium mechanics and its numerical approximation, European Journal of Mechanics, A/Solids.

Rogulja D, Rauskolb C and Irvine KD (2008) Morphogen Control of Wing Growth through the Fat Signaling Pathway, Developmental Cell, 15: 309-321.

Schluckl T, Aegerter CM,(2010) Photo-elastic properties of the wing imaginal disc of Drosophila. Eur.Phys.J.: 111-115.

Shraiman BI, Mechanical feedback as a possible regulator of tissue growth (2005) Proc. Natl Acad. Sci. USA 102: 3318-3323.

Wartlick O, Mumcu P, Kicheva A, Bittig T, Seum C, Jülicher F and Gonzalez-Gaitan M (2011a) Dynamics of Dpp signaling and proliferation control, Science, 331: 1154-1159

Wartlick O, Mumcu P, Jülicher F and Gonzalez-Gaitan M (2011b) Understanding morphogenetic growth control - lessons from flies, Nature Reviews Molecular Cell Biology, 12:594-604

Weigmann K, Klapper R, Strasser T, Rickert C, Technau G, Jckle H, Janning W and Klmbt C (2003) 
FlyMove - a new way to look at development of Drosophila, Trends Genet 19, 310. 